\title{
Formulation and Pharmacokinetic Evaluation of Tetracycline-Loaded Solid Lipid Nanoparticles for Subcutaneous Injection in Mice
}

\author{
Xi-ming XU, ${ }^{* a} a$ Yan-song WANG ${ }^{a}$ Rong-ying Chen, ${ }^{a}$ Chun-lai Feng, ${ }^{b}$ Feng YAO, ${ }^{c}$ Shan-shan Tong, ${ }^{a}$ \\ Li Wang, ${ }^{a}$ Fumiyoshi Yamashita, ${ }^{b}$ and Jiang-nan YU*,a \\ ${ }^{a}$ Department of Pharmaceutics, School of Pharmacy, Jiangsu University; Zhenjiang 212013, P. R. China: ${ }^{b}$ Department of \\ Drug Delivery Research, Graduate School of Pharmaceutical Sciences, Kyoto University; Kyoto 606-8501, Japan: and \\ ${ }^{c}$ Department of Surgery, Harvard Medical School, Brigham and Women's Hospital; Massachusetts 02115, U.S.A. \\ Received September 29, 2010; accepted November 27, 2010; published online December 1, 2010
}

The aim of this work was to prepare tetracycline-loaded solid lipid nanoparticles (Tet-SLN), and to evaluate the potential of these colloidal carriers for subcutaneous injection. Tet-SLN was prepared by microemulsion method and the preparation conditions were optimized by ternary phase diagram. At optimized process conditions, lyophilized Tet-SLN showed spherical particles with a mean diameter of $87.2 \pm 46.9 \mathrm{~nm}$ and a negative zeta potential of $-6.69 \mathrm{mV}$, up to $1.7 \%$ tetracycline drug content was achieved after loading. In vitro release test showed a biphasic release profile for Tet-SLN and more than $80 \%$ of the drug was liberated from Tet-SLN in $48 \mathrm{~h}$. After subcutaneous injection of Tet-SLN to mice, a considerable sustained release was observed; tetracycline in blood could be detected lasting $36 \mathrm{~h}$, and lower concentrations of tetracycline in all tissues tested compared to the free tetracycline solution were observed. In conclusion, Tet-SLN can be prepared well by microemulsion method and subcutaneous injection of SLN provide a new perspective for drug sustained release.

Key words tetracycline; solid lipid nanoparticle; microemulsion technique; ternary phase diagram; biodistribution

The tetracyclines are broad-spectrum antimicrobial agents which inhibit protein synthesis by binding to the $30 \mathrm{~S}$ ribosomal subunit. They exhibit activity against many Gram-positive and Gram-negative bacteria, Mycoplasma, Chlamydia and Rickettsiae, as well as spirochetes and some parasites. ${ }^{1,2)}$ Traditionally tetracyclines have been used for the treatment of a variety of infections including atypical pneumonia, diarrheal diseases, and periodontal diseases. ${ }^{2)}$ Recent years, the additional application besides antimicrobial effects of tetracyclines has been discovered and developed. For example, tetracyclines contribute to the impaired collagen biosynthesis and inhibition of matrix metalloproteases (MMPs) indicating their potential use in the treatment of diabetes and osteoarthritis. ${ }^{3,4)}$ However, drug-resistance, a common problem confronted by almost all antibiotics, and side effects accompanied with long-term administration of tetracyclines limit their use in many infectious diseases and other area. ${ }^{1,2)}$

Sustained-release drug delivery systems are regarded as promising formulations to improve therapeutic efficacy and safety of drugs by delivering them at a rate dictated by the need of the physiological environment over a period of treatment to the site of action. The most often used sustainedrelease drug delivery systems include hydrogels, microspheres, liposomes, micelles, nanoparticles, and nanocrystals. Among the various sustained-release drug delivery systems, solid lipid nanoparticles (SLN) are attracting major attention due to their unique advantages, such as good tolerability, larger scale production, possibility of controlled released targeting, less acute and chronic toxicity, and avoidance of toxic organic solvents. ${ }^{5,6}$ )

So far, a few tetracyclines sustained release formulations such as, microspheres, ${ }^{7)}$ hydrogel, ${ }^{8)}$ liposome ${ }^{9)}$ and fiber ${ }^{10}$ have been developed. Although SLN sustained release drug delivery system has many advantages compared to these systems, no tetracycline-loaded SLN has been reported to our knowledge.

On the other hand, different routes of administration may result in varying effects on the biodistribution pattern of drug carriers. $^{5,11)}$ The pharmacokinetics and biodistribution characteristics of drug encapsulated SLN have been well characterized and compared following intravenous (i.v.) injection, ${ }^{12-14)}$ and oral (per os (p.o.)) administration. However, few attempts have been done on the biodistribution of drug encapsulated SLN after subcutaneous (s.c.) administration. ${ }^{15,16)}$

The aim of this work was to prepare tetracycline-loaded solid lipid nanoparticles (Tet-SLN), and to investigate the biodistribution of Tet-SLN after subcutaneous administration in mice.

\section{Results and Discussion}

Construction of Ternary Phase Diagrams The prerequisite to obtain a sufficient loading capacity is a sufficiently high solubility of the drug in the lipid melt. Albeit tetracycline is poorly soluble in lipids used in the study, it is soluble in ethanol. Besides, both glyceryl monostearate (GM) and stearic acid (SA) well dissolve in ethanol; therefore, absolute ethanol was used as the co-solvent in order to obtain a homogenous drug-lipids mixture which would facilitate the formation of microemulsion and the final SLN product. After the formation of a uniform drug-lipids mixture, ethanol was completely removed by evaporation under reduced pressure. The area of microemulsion is depicted in the phase diagrams indicated by shaded color (Figs. 1a-e).

In all cases, initiation of microemulsion formation started as a clear viscous phase (gel microemulsion) which further led to the formation of clear liquid microemulsion upon subsequent dilution with aqueous phase. Ternary phase diagrams show that $K_{\mathrm{m}}$ values $\left(K_{\mathrm{m}}=\mathrm{GM}: \mathrm{SA}\right.$ by weight) within the range of $2: 3$ to $4: 1$ (included) were much desirable as indicated by larger emulsification area of Figs. 1b-d compared with that of others. Moreover, it was found that the freezedried SLN was scarcely redispersed in aqueous phase when $K_{\mathrm{m}}$ value was less than $4: 1$, while the microemulsion was 

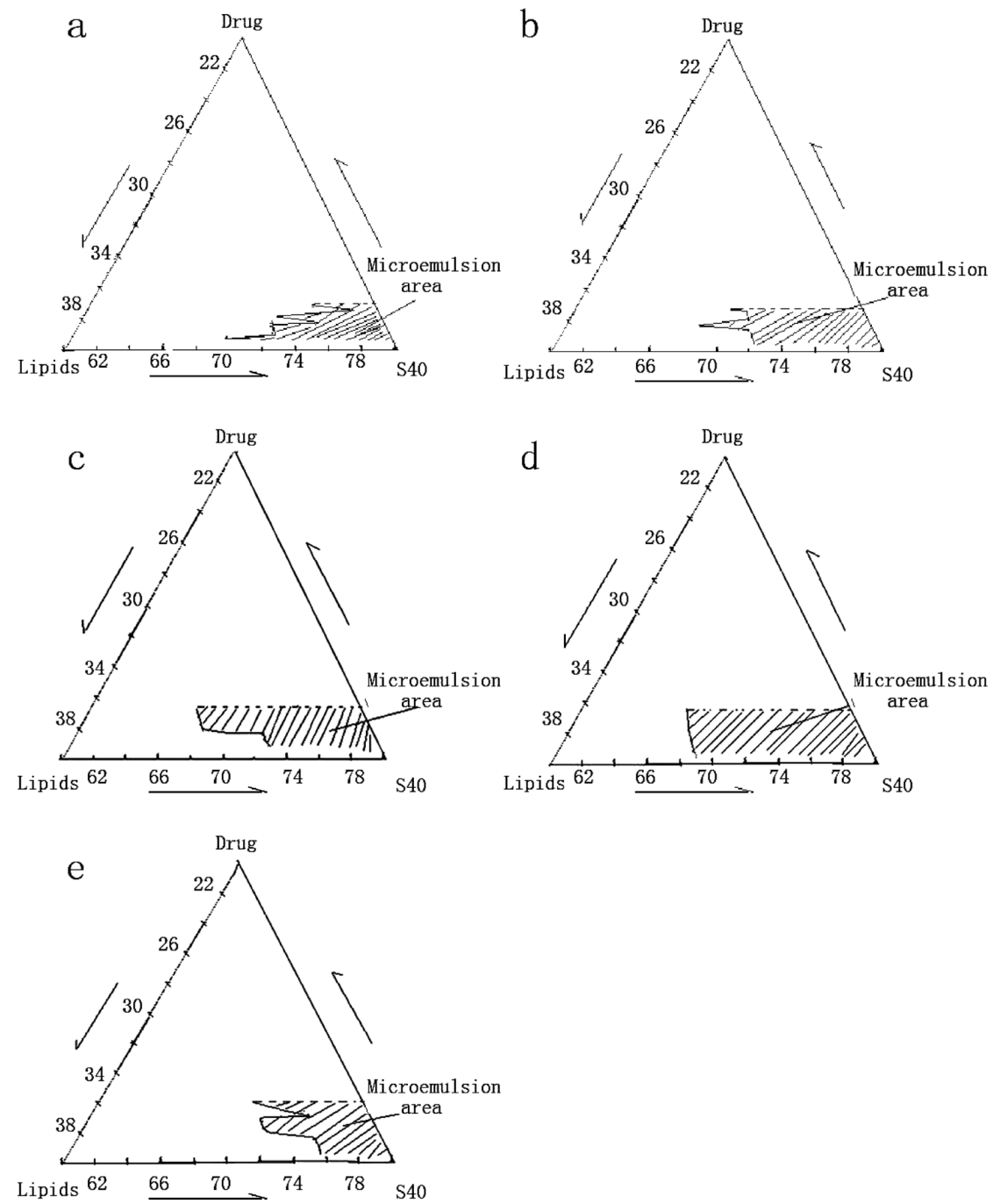

Fig. 1. Ternary Phase Diagrams of Durg-Surfactant-Lipids System at Different $K_{\mathrm{m}}\left(K_{\mathrm{m}}=\mathrm{GM}: \mathrm{SA}, \mathrm{w} / \mathrm{w}\right)(5: 0$ (a), $4: 1$ (b), $2: 3$ (c), $3: 2$ (d), $1: 4$ (e)) at $59 \pm 1{ }^{\circ} \mathrm{C}$

The shaded region represents microemulsion area.

inclined to layer and precipitate upon storage over one day when the $K_{\mathrm{m}}$ value was less than $3: 2$. Based on these experimental results, optimal SLN which resulted in larger microemulsion area and also exhibited excellent microemulsion stability and redispersibility were obtained when the $K_{\mathrm{m}}$ value was $4: 1$. To see whether the surfactants in the aqueous phase can effect the formation of microemulsion, aqueous phase containing various surfactants including Poloxamer 188, Poloxamer 407, $\mathrm{NaCl}$, Tween 80 , sodium cholate and sodium dodecyl sulfate (SDS) was investigated. From phase diagram, it could be found that surfactants in the aqueous phase neither significantly enlarged the microemulsion region nor improved the stability of microemulsion (data are not shown). Therefore, double distilled water without any surfactant was used as the aqueous phase for producing microemulsion. The optimum amount of water added, $20 \mathrm{ml}$ was found when forming a homogenous and transparent microemulsion in this study. In all cases, drug content was fixed at $3 \%$, a limit beyond which the drug would precipitate from the microemulsion despite any efforts of adjusting composition of ingredients. Under optimized conditions, SLN could be successfully prepared with GM, SA and S-40 in a
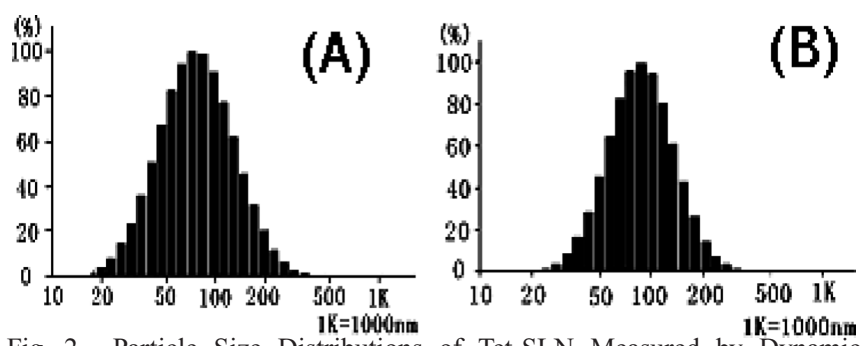

Fig. 2. Particle Size Distributions of Tet-SLN Measured by Dynamic Light Scattering

A: SLN-1 (with $\mathrm{NaCl}$ ); B: SLN-2 (without $\mathrm{NaCl}$ ).

reproducible manner without any agglomeration.

Characterization of Tet-SLN It has been reported that types and concentration of cryoprotectants exert effects on the particle sizes and size distribution of SLN. ${ }^{17)}$ In this study, solution of $2 \%$ mannitol and the mixture solution of $1 \% \mathrm{NaCl}$ and $1 \%$ mannitol were selected as the cryoprotective agents and their effects on the particle sizes and zeta potentials were studied. Figure 2 shows the size distributions of SLN prepared using mannitol as cryoprotective agents with or without $\mathrm{NaCl}$. More detailed results were listed in Table 1. 
Table 1. Effect of Lyoprotectant on Size and Zeta Potential of SLN

\begin{tabular}{|c|c|c|c|}
\hline Formulation & $\begin{array}{l}\text { Lyoprotectant in } \\
\text { theaqueous phase }\end{array}$ & Size & $\begin{array}{c}\text { Zeta } \\
\text { potential }\end{array}$ \\
\hline SLN-1 & $2 \%$ mannitol & $95.8 \pm 43.3 \mathrm{~nm}$ & $-6.69 \mathrm{mV}$ \\
\hline SLN-2 & $1 \% \mathrm{NaCl}$ and $1 \%$ mannitol & $87.2 \pm 46.9 \mathrm{~nm}$ & $-0.23 \mathrm{mV}$ \\
\hline
\end{tabular}

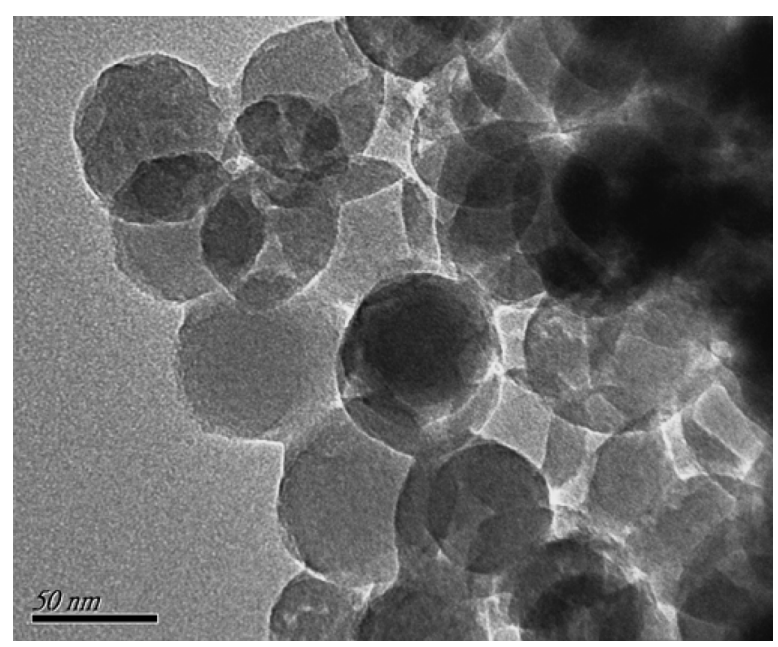

Fig. 3. TEM Micrograph of Tet-SLN Prepared under the Optimal Conditions with $\mathrm{NaCl}$

Figure 3 shows the transmission electron microscopy (TEM) micrograph of SLN prepared using mannitol as cryoprotective agents with $\mathrm{NaCl}$.

As can be seen from Fig. 3, the SLN obtained were near spherical in shape and approximately homogeneous in sizes. As demonstrated in Table 1 and Fig. 2, the addition of $\mathrm{NaCl}$ in the aqueous phase as the co-lyoprotectant reduced the average size of SLN, and resulted in a smaller size distribution, and decreased the zeta potentials. Considering that zeta potentials of larger absolute value would be more favorable for maintaining a stable colloidal system, ${ }^{18)}$ the $2 \%$ of mannitol, which resulted in higher zeta potentials, was preferred as the lyoprotectant for the preparation of Tet-SLN.

The regression equation of tetracycline was $\mathrm{A}=34.806 \mathrm{C}-$ $0.0232(n=7, r=0.9998)$ and based on this formula, the drug content was calculated to be $1.73 \%$.

The changes in the particle size and incorporation efficiency of SLN are widely used as indicators of storage stability, ${ }^{19,20)}$ In most cases, an increase of particle size was the result of gelation while decease of incorporation efficiency was caused by drug expulsion during the storage time. ${ }^{5}$ In this research, we both studied the stability of SLN powder and dispersion. SLN dispersion is a thermodynamically unstable heterogeneous phase system, tending to aggregate and layer after long term storage. Accordingly, the time it takes for layering can be used as a convenient indicator for the stability of SLN dispersion. The centrifugation method which is originally used for the stability study of emulsions was modified and applied for the stability test of SLN dispersion. After centrifugation at $3000 \mathrm{rpm}$ for $15 \mathrm{~min}$, Tet-SLN dispersion did not layer, nor drug precipitated, showing a favorable stability. As to lyophilized SLN powder and aqueous suspension stored at $25^{\circ} \mathrm{C}$, the color of both samples

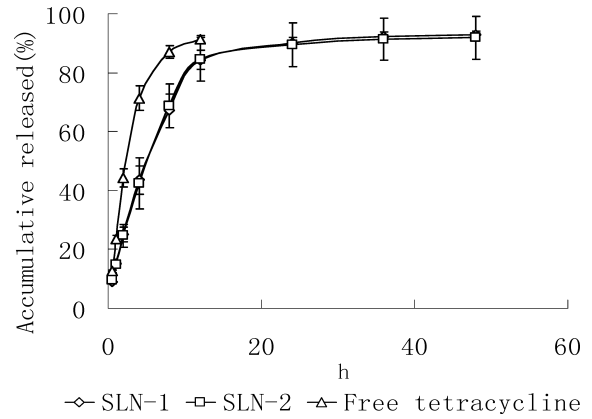

Fig. 4. In Vitro Release Profiles of SLN-1, SLN-2 and Free Tetracycline

Table 2. Release Equations of SLN-2

\begin{tabular}{lcc}
\hline \hline \multicolumn{1}{c}{ Model } & Equation & $\begin{array}{c}\text { Correlation } \\
\text { coefficient }(r)\end{array}$ \\
\hline First order & $\ln (1-\mathrm{Q})=-0.0534 t-0.4539$ & 0.9012 \\
Higuchi & $Q=0.1407 t^{1 / 2}+0.1177$ & 0.9131 \\
Hixcon Crowell & $(1-Q)^{1 / 3}=0.009 t+0.6539$ & 0.7549 \\
Nibergull & $(1-Q)^{1 / 2}=0.0117 t+0.54$ & 0.7702 \\
Weibull & $\ln \ln (1 /(1-Q))=0.7633 \ln t-1.6933$ & 0.9830 \\
\hline
\end{tabular}

$Q=M_{t} / M_{\infty}$, where $M_{t}$ is the cumulative amount of drug released in time $t$ and $M_{\infty}$ is the total amount of drug released.

changed from yellow to dark pink within one week, and precipitation of particles observed. Furthermore, the SLN suspension stored in the refrigerator also precipitated. By contrast, the lyophilized SLN powder stored in the refrigerator could maintain stable over one month with a slight increase in the size (from 95.8 \pm 43.3 to $109.8 \pm 59.3 \mathrm{~nm}$ ). No apparent change in the morphology and redispersibility was observed.

In Vitro Release Figure 4 shows the release profile of tetracycline from the SLN-1, SLN-2 (SLN-1: freeze dried with $\mathrm{NaCl}$; SLN-2: freeze dried without $\mathrm{NaCl}$ ) and the free tetracycline solution. The similar release behavior of SLN-1 and SLN-2 indicates that the addition of $\mathrm{NaCl}$ as the colyoprotectant did not affect the release profile of SLN. Biphasic release profile was observed for Tet-SLN which demonstrated by the initial burst release within $10 \mathrm{~h}$ was followed by a prolonged release. More than $80 \%$ of tetracycline was released from the SLN over $48 \mathrm{~h}$. The in vitro drug release equations of SLN-2 were listed in Table 2. The data revealed that the sustained release profile fitted best to Weibull equation. The drug encapsulation patterns in these particles, (i.e., matrix type or reservoir type) and surface properties could both affect the release behavior of this SLN. Based on the observed apparent initial drug release, it could be deduced that our Tet-SLN was a core-shell structure, and tetracycline was enriched in the shell. Tetracycline incorporated in the shell released rapidly, tetracycline loaded in the core could only be released slowly from the lipid matrices through dissolution and diffusion. ${ }^{21-23)}$

Biodistribution Study The present study clearly demonstrates the sustained release of tetracycline from s.c. administrated Tet-SLN. Figure 5A shows the drug concentration in the injection site after s.c. administration of free tetracycline and Tet-SLN. Both Tet-SLN and free drug were eliminated quickly from the administration site. The tetracycline was almost undetectable $6 \mathrm{~h}$ post-injection of tetracycline and no drug could be detected after $8 \mathrm{~h}$. The $A U C_{0-\tau}$ and mean resi- 

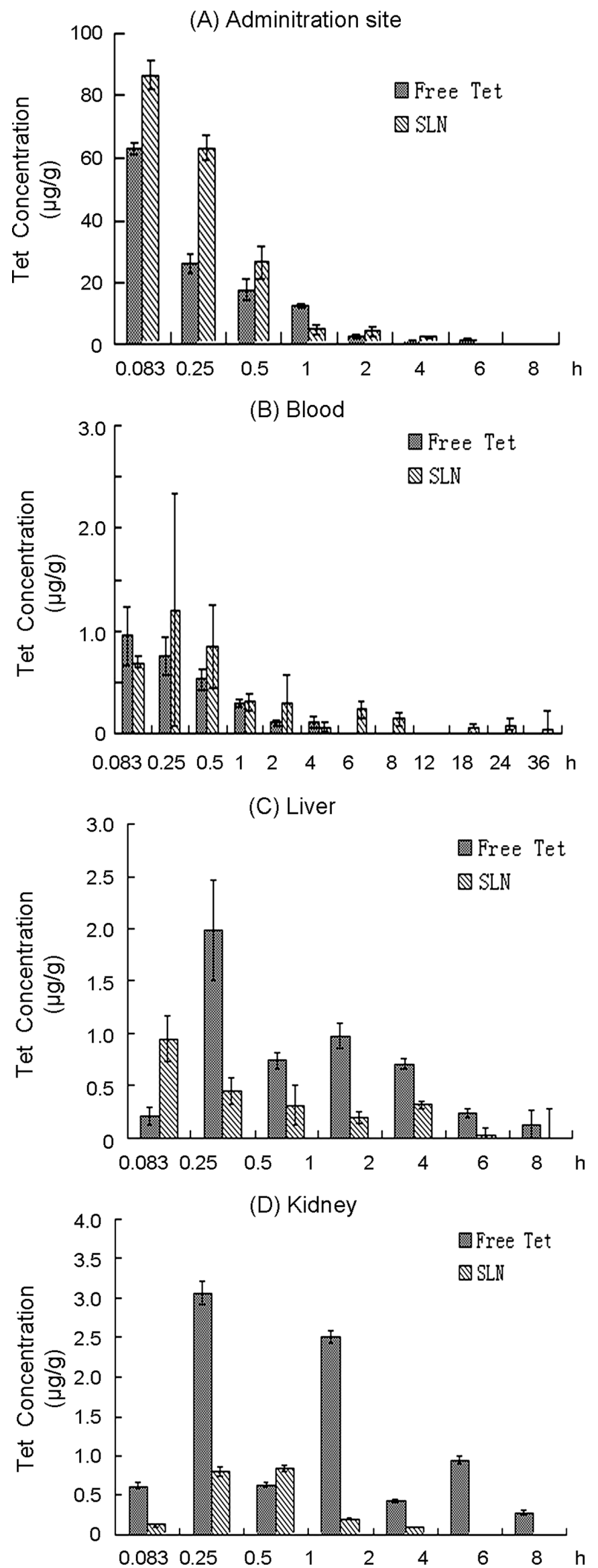

Fig. 5. Distribution Diagrams of Tetracycline in the Administration Site, Blood, Liver and Kidney $(n=5)$ dence time $(M R T)$ in the injection area for free drug and SLN suspension were $35.1 \mathrm{~h} \mu \mathrm{g} / \mathrm{ml}, 1.0 \mathrm{~h}$ and $48.5 \mathrm{~h} \mu \mathrm{g} / \mathrm{ml}, 1.1 \mathrm{~h}$, respectively. After subcutaneously injection, free tetracycline was rapidly delivered into the interstitial area underlying the dermis of the skin and was absorbed preferentially and quickly by the blood capillaries. ${ }^{24)}$ Tetracycline detected in the blood $5 \mathrm{~min}$ (namely $0.083 \mathrm{~h}$ ) after injection confirmed its rapid absorption from the injection site to the blood circulation. Tet-SLN demonstrated a rapid elimination from the injection site with higher drug concentrations in the first half hour due to a slower diffusion rate as of nanoparticles compared with drug molecules. The results are inconsistent with the literatures ${ }^{15,16)}$; their results showed SLN formulation of drugs could remain in the injection site for a long time. We consider the lymphatic systems might be responsible for the diffusing route of particulates with size around $100 \mathrm{~nm}$, it is, therefore, more likely for Tet-SLN to be absorbed by the lymphatics than by the blood capillaries. ${ }^{24-27)}$

The blood clearance profiles differed dramatically following s.c. administration of encapsulated and free tetracycline as shown in Fig. 5B. The $A U C_{0-\tau}$ and $M R T$ in the blood for free drug and SLN suspension were $0.53 \mathrm{~h} \mu \mathrm{g} / \mathrm{ml}, 10.4 \mathrm{~h}$ and $4.12 \mathrm{~h} \mu \mathrm{g} / \mathrm{ml}, 44.1 \mathrm{~h}$, respectively. After injection of free drug, an initial peak level in the blood followed by a quick decline had been expected as the drug concentration at the injection site remarkably diminished. ${ }^{28)}$ However, the blood level of tetracycline had been detectable for merely $4 \mathrm{~h}$ and it was keeping declining, suggesting a very fast drug clearance from the blood circulation. Moreover, higher concentration of tetracycline in kidney and liver compared with Tet-SLN also indicates its rapid blood clearance. Comparatively, higher and longer drug concentration was attained over $36 \mathrm{~h}$ following s.c. administration of the Tet-SLN, indicating a contribution of the controlled release capacity of SLN. As tetracycline was controllably released into the blood, it was quickly distributed into other tissues and organs within a short time, resulting in a declining drug concentration following peak level at $0.25 \mathrm{~h}$. To our knowledge, it is the first time that a controlled drug release pattern over $36 \mathrm{~h}$ from subcutaneously administered SLN has been reported, following studies that observed $6 \mathrm{~d}$ of detectable drug levels after s.c. delivery of liposomes reported by Postma et al. ${ }^{28)}$

Figures $5 \mathrm{C}$ and D shows that the s.c. delivery of Tet-SLN resulted in a much lower drug distribution in liver and kidney, which maintained only for $2 \mathrm{~h}$. According to literature, tetracycline is widely distributed throughout the body and tissue secretions with relative higher accumulations in the liver and kidney and is excreted mainly via urine. ${ }^{1,29)}$ In this study, after administration of free drug, tetracycline in lower but detectable concentration (data not shown) was observed in organs including heart, brain, stomach, spleen and lung, whereas comparatively higher drug accumulation in liver and kidney was detected in agreement with previous reports. However, post-injection of Tet-SLN, drug levels in organs including heart, spleen, lung and brain were almost undetectable except liver and kidney, demonstrating that subcutaneous administration of Tet-SLN greatly reduced drug accumulation in organs especially liver and kidney, which can confer to a potential decrease of hepatotoxicity and nephrotoxicity. ${ }^{2)}$ Our result is in accordance with previous studies about the reduced tissue redistribution following s.c. admin- 
istration of SLN or liposomes compared with intravenous or intraperitoneal administration. ${ }^{30)}$ Apart from the administration route, particle size is another decisive factor that affects the in vivo drug distribution. ${ }^{31,32)}$ Not only particles with large size are more likely to localize at the s.c. injection site, but the drug vehicles with diameters larger than $200 \mathrm{~nm}$ are reportedly prone to be scavenged non-specifically by monocytes and the reticuloendothelial system (RES), resulting in a significant decrease in plasma half-life and a higher drug concentration in organs such as lung, liver and spleen. ${ }^{32-34)}$ Conversely, particles less than $150 \mathrm{~nm}$ tend to avoid macrophage uptake and allow for a higher drug concentration with longer blood circulation time and lower biodistribution in RES organs. ${ }^{31,32)}$ Therefore, tetracycline encapsulated SLN with size less than $150 \mathrm{~nm}$ were quickly absorbed from the injection site after s.c. administration, extended drug duration in the blood and extensively lowered drug accumulation in tissues.

\section{Conclusion}

Although the use of solid lipid nanoparticles in medicine and more specifically drug delivery is set to spread rapidly, the data on the administration route of SLN are still scares. In this study, we focus on the bioavailability and biodistribution of Tet after subcutaneous Tet-SLN. In our work, after subcutaneous administration of Tet-SLN, prolonged drug release in the blood circulation was obtained, however, no drug accumulation at the injection site was observed. The results are inconsistent with the literatures. ${ }^{16,17)}$ In a word, the sustained release of tetracycline from the SLN in the blood circulation following subcutaneous administration offered a new perspective among various pharmaceutical efforts to extend the drug detainment in blood circulation. Lower tissue redistribution of tetracycline after subcutaneous injection of Tet-SLN may help to decrease side affects.

\section{Experimental}

Reagents Tetracycline (Tet) was purchased from Sigma-Aldrich Co., Ltd., China. GM was obtained from Shanghai Hengxin Chemical Reagent Co., Ltd., China. SA was purchased from Sinopharm Chemical Reagent Co., Ltd., Shanghai, China, and Polyoxyl 40 stearate (S-40) was purchased from Nanjing Well Chemical Co., Ltd., China. Double distilled water was used throughout. All other chemicals and solvents were HPLC or analytical grade.

Preparation of Tet-SLN by Microemulsion Technique Tet-SLN was prepared by microemulsion technique. Briefly, $2.0 \mathrm{~g}$ of S-40, $0.64 \mathrm{~g}$ of GM, $0.16 \mathrm{~g}$ of SA and $60 \mathrm{mg}$ of tetracycline were dissolved in $10 \mathrm{ml}$ of absolute alcohol. Then the alcohol was completely evaporated $59 \pm 1{ }^{\circ} \mathrm{C}$ using a rotary evaporator (Heidolph, Germany) to obtain a homogenous drug-contained oil phase. Twenty milliliters of double distilled water $\left(59 \pm 1^{\circ} \mathrm{C}\right)$ was added dropwise into the oil phase under stirring (Electric Agitator, China) at $400 \mathrm{rpm}$ for $15 \mathrm{~min}$, resulting in a transparent and thermodynamically stable gel microemulsion system. Ten milliliters of $2 \% \mathrm{w} / \mathrm{v}$ ice-cold mannitol solution was immediately added dropwise to the obtained microemulsion under stirring at $800 \mathrm{rpm}$ for $20 \mathrm{~min}$ in icy water bath. The resultant solution was lyophilized (Freeze Drier, Christ, Germany) for $48 \mathrm{~h}$ to obtain SLN powders, which were later stored at $4{ }^{\circ} \mathrm{C}$, and protected from light and moisture before use.

Construction of Ternary Phase Diagrams The ternary phase diagrams were constructed by titration of homogenous liquid mixtures of lipid, emulsifier and aqueous phase containing surfactants. SA and GM at different $K_{\mathrm{m}}$ values and S-40 are used as the oil phase and emulsifier, respectively.

At different $K_{\mathrm{m}}$ values of $5: 0,4: 1,3: 2,2: 3$, and $1: 4$, the required quantities of SA, GM, S-40 and tetracycline were mixed in the alcohol at $59 \pm 1{ }^{\circ} \mathrm{C}$ to obtain homogenous oil phase. The oil phase was slowly titrated with aliquots of distilled water $\left(59 \pm 1^{\circ} \mathrm{C}\right)$ containing surfactants and stirred until equilibrium was reached. The transparent mixtures were further titrated with aliquots of distilled water until they became turbid. The clear and transparent samples were considered being within the microemulsion region. No attempts were made to completely identify the other regions of the phase diagrams. Based on properties including the area of microemulsion, the stability of microemulsion at $25^{\circ} \mathrm{C}$ and the redispersibility of lyophilized SLN, we selected the most appropriate ratio of lipids, drug content and aqueous phase for the preparation of Tet-SLN.

Characterization of Tet-SLN Transmission electron microscopy (JEM2100, JEOL) was employed to determine the shape and morphology of TetSLN. The mean particle sizes and zeta potentials of Tet-SLN were determined by dynamic light scattering method using Nicomp 380 ZLS particle sizer (PSS, U.S.A.) after resuspending of lyophilized SLN in water. Each measurement was carried out in triplicate.

Shaking method was used to assess the redispersibility of Tet-SLN. Simply, a volume of water was added in the lyophilized Tet-SLN until a fully redispersion after shaking was observed.

Tet-SLN $(30 \mathrm{mg})$ was precisely weighed and dissolved in $8 \mathrm{ml}$ of absolute ethanol at $60^{\circ} \mathrm{C}$ to dissolve lipid matrices. Then, the solution was vortexmixed for $3 \mathrm{~min}$ before centrifugation (Refrigerated centrifuge, Biofuge Stratos Hereaus, Germany) at $3000 \mathrm{rpm}$ for $10 \mathrm{~min}$. The supernatant was diluted and filtrated through $0.45 \mu \mathrm{m}$ microporous membrane for the determination of tetracycline by UV spectrophotometry at $358 \mathrm{~nm}$ (Shimadzu UV2401 PC Spectrophotometer, Japan). Free tetracycline solution was treated with the same method as a reference. Each determination was carried out in triplicate. The drug content was calculated according to the following formula:

drug content $=($ actual drug weight $/$ weight of drug loaded SLN $) \times 100 \%$

The stability of Tet-SLN was investigated in two aspects: first, an aliquot of Tet-SLN suspension was centrifuged at $3000 \mathrm{rpm}$ for $15 \mathrm{~min}$ to check whether or not the solution would layer; second, stored both lyophilized TetSLN powder and Tet-SLN suspension at $25^{\circ} \mathrm{C}$ and in a refrigerator at $4{ }^{\circ} \mathrm{C}$ to observe changes in the morphology, redispersibility and particle size of Tet-SLN at predetermined time. All experiments were conducted in triplicate.

In Vitro Release Study In vitro release of tetracycline from Tet-SLN was investigated by dialysis method with dialysis membrane (CAS[MD253500]) against $0.1 \mathrm{~m}$ phosphate buffer solution (PBS, pH 7.4, containing $0.02 \%$ sodium azide). Briefly, the Tet-SLN were suspended with double distilled water in a dialysis bag and then put in a $15 \mathrm{ml}$ of PBS as the release medium under shaking at $100 \mathrm{rpm}$ and $37^{\circ} \mathrm{C}$ using shaking gas bath (Jiangsu Jintan Medical Instrument Factory, China). Aliquots of dialysate sample were withdrawn at predetermined time and replaced immediately with the same volume of fresh PBS. The concentration of tetracycline released at each time point was determined by UV spectrophotometry at $358 \mathrm{~nm}$. Free tetracycline solution was treated in the same way and its release medium was used as the blank. All measurements were performed in triplicate.

In Vivo Study Male Kunming strain mice $(22 \pm 2 \mathrm{~g})$ were supplied by Qinglong Mountain Animals Breeding Field in Jiangning District, Nanjing, China. The mice were randomly divided into experimental group and control group, and were given food and water without tetracycline. For the experimental group, suspension of Tet-SLN was subcutaneously administrated at the left foot pad of each mouse with the dosage of $1.25 \mu \mathrm{g} / \mathrm{g}$. Similarly, free tetracycline suspension was given to another group of mice as the control group. At known time intervals, mice were sacrificed and the blood was collected in heparinized tubes, then centrifuged at $5000 \mathrm{rpm}$ for $10 \mathrm{~min}$ to obtain plasma samples. The organs including heart, liver, spleen, lung, kidney, stomach, brain and the left foot pad were dissected and precisely weighed. All samples were stored at $-20^{\circ} \mathrm{C}$ till pretreatment. The experimental protocol was approved by the University Ethics Committee for the use of experimental animals and conformed to the Guide for Care and Use of Laboratory Animals.

In the sample preparation for HPLC analysis, oxytetracycline-methanol solution $(5 \mu \mathrm{g} / \mathrm{ml})$ was used as the internal standard. Briefly, $20 \mu \mathrm{l}$ of methanol, $20 \mu \mathrm{l}$ of the internal standard, $30 \mu \mathrm{l}$ of $1 \% \mathrm{H}_{3} \mathrm{PO}_{4}, 100 \mu \mathrm{l}$ of PBS $(\mathrm{pH} 4.0)$ and $5 \mathrm{ml}$ of mixed solution of ethyl acetate and cyclohexane $(3: 1$, $\mathrm{v} / \mathrm{v}$ ) were sequentially added into the plasma and homogenized tissue samples. Then all samples were vortex-mixed for $5 \mathrm{~min}$ and centrifuged at $3000 \mathrm{rpm}$ and $4{ }^{\circ} \mathrm{C}$ for $10 \mathrm{~min}$. After centrifugation, $4 \mathrm{ml}$ of the supernatant was collected and evaporated to dryness under a current of nitrogen gas at $35^{\circ} \mathrm{C}$. Finally, the dried residue was reconstituted with $100 \mu 1$ of the mobile 
phase (acetonitrile-methanol-5\% acetic acid $(7.4: 9: 83.6, \mathrm{v} / \mathrm{v})$ and ethylenediaminetetraacetic acid (EDTA) $\mathrm{Na}_{2}(0.6 \mathrm{~mm}), \mathrm{pH} 2.9$ adjusted with triethylamine) and then $20 \mu \mathrm{l}$ of the reconstitution was injected into the HPLC system (Shimadzu, Japan) equipped with an SCL-10Avp system controller, LC-10ADvp pump, SPD-M10Avp UV/Vis-PDA detector. Tetracycline in samples was separated using a Simpak C18 $(150 \mathrm{~mm} \times 4.6 \mathrm{~mm})$ chromatographic column at $1.0 \mathrm{ml} / \mathrm{min}$ flow rate and detected at the wavelength of $358 \mathrm{~nm}$. Peak recording and integration were performed using Shimadzu CLASS-VP data process system. Peak area ratios of the tetracycline and the internal standard were used for quantitative analysis.

Statistical Analysis The pharmacokinetic data were calculated with the pharmacokinetics program BAPP 3.0 software (China Pharmaceutical University) and all data are expressed as mean \pm S.D.

Acknowledgements The Project Sponsored by the Scientific Research Foundation for the Returned Overseas Chinese Scholars, State Education Ministry and by the Special Foundation for the Training of Six High-Tech Talent in Jiangsu Province (No. 07-c-012).

\section{References}

1) Albarellos G. A., Landoni M. F., Vet. J., 180, 304-316 (2009).

2) Epstein M. E., Groton M. A., Sadick N. S., J. Am. Acad. Dermatol., 37, 365-384 (1997).

3) Karna E., Palka J., Wolczynski S., Eur. J. Pharmacol., 430, 25-31 (2001).

4) Craig R. G., Yu Z., Xu L., Barr R., Ramamurthy N., Boland J., Schneir M., Golub L. M., Biochim. Biophys. Acta, 1402, 250-260 (1998).

5) Mehnert W., Mäder K., Adv. Drug Deliv. Rev., 47, 165-196 (2001).

6) Müller R. H., Mäder K., Gohla S., Eur. J. Pharm. Biopharm., 50, $161-177$ (2000).

7) Mundargi R. C., Srirangarajan S., Agnihotri S. A., Patil S. A., Ravindra S., Setty S. B., Aminabhavi T. M., J. Controlled Release, 119, 5968 (2007).

8) Singh B., Chauhan N., Kumar S., Bala R., Int. J. Pharm., 352, 74-80 (2008).

9) Sangaré L., Morisset R., Omri A., Ravaoarinoro M., J. Antimicrob. Chemoth., 42, 831-834 (1998).

10) Kenawy E.-R., Bowlin G. L., Mansfield K., Layman J., Simpsonc D. G., Sanders E. H., Wnek G. E., J. Controlled Release, 81, 57-64 (2002).

11) Gaumet M., Vargas A., Gurny R., Delie F., Eur. J. Pharm. Biopharm., 69, $1-9$ (2008).

12) Sharma P., Ganta S., Denny W. A., Garg S., Int. J. Pharm., 367, 187-
194 (2009).

13) Ye J. S., Wang Q., Zhou X. F., Zhang N., Int. J. Pharm., 352, 273-279 (2008).

14) Huang G. H., Zhang N., Bi X. L., Dou M. J., Int. J. Pharm., 355, $314-320$ (2008).

15) Reddya L. H., Sharmab R. K., Chuttanib K., Mishrab A. K., Murthy R. S. R., J. Controlled Release, 105, 185-198 (2005).

16) Lu B., Xiong S., Yang H., Yin X., Chao R., Eur. J. Pharm. Sci., 28, $86-95$ (2006).

17) Zimmermann E., Müller R. H., Mäder K., Int. J. Pharm., 196, $211-$ $213(2000)$.

18) Gokce E. H., Sandri G., Bonferoni M. C., Rossi S., Ferrari F., Güneri T., Caramella C., Int. J. Pharm., 364, 76-86 (2008).

19) Venkateswarlu V., Manjunath K., J. Controlled Release, 95, 627-638 (2004).

20) Liu J., Gong T., Wang C. G., Zhong Z. R., Int. J. Pharm., 340, 153162 (2007).

21) Zur Muhlen A., Schwarz C., Mehnert W., Eur. J. Pharm. Biopharm., 45, 149-155 (1998).

22) Zur Muhlen A., Mehnert W., Pharmazie, 53, 552—555 (1998).

23) Grassia M., Voinovichb D., Franceschinisb E., Perissuttib B., FilipovicGrcic J., J. Controlled Release, 92, 275-289 (2003).

24) McLennan D. N., Porter C. J. H., Charman S. A., Drug Discov. Today: Technologies, 2, 89-96 (2005).

25) Wilson K. D., Raney S. G., Sekirov L., Chikh G., deJong S. D., Cullis P. R., Tam Y. K., Int. Immunopharmacol., 7, 1064-1075 (2007).

26) Oussoren C., Storm G., Adv. Drug Deliv. Rev., 50, 143-156 (2001).

27) Oussoren C., Zuidema J., Crommelin D. J. A., Storm G., Biochim. Biophys. Acta, 1328, 261-272 (1997).

28) Postma N. S., Boerman O. C., Oyen W. J. G., Zuidema J., Storm G., J. Controlled Release, 58, 51-60 (1999).

29) Marzo A., Dal Bo L., J. Chromatogr. A, 812, 17-34 (1998).

30) Fundarò A., Cavalli R., Bargoni A., Vighetto D., Zara G. P., Gasco M. R., Pharm. Res., 42, 337-343 (2000).

31) Neubergera T., Schöpf B., Hofmann H., Hofmann M., von Rechenberg B., J. Magn. Magn. Mater, 293, 483-496 (2005).

32) Yang S. C., Lu L. F., Cai Y., Zhu J. B., Liang B. W., Yang C. Z., J. Controlled Release, 59, 299-307 (1999).

33) Yang X. G., Li L. B., Wang Y. X., Tan Y. B., Int. J. Pharm., 370, 210 215 (2009).

34) Ma H. L., Xu Y. F., Qi X. R., Maitani Y., Nagai T., Int. J. Pharm., 354, $217-226$ (2008). 